\title{
Medición de la calidad de vida en médicos residentes
}

\author{
S.E. Prieto-Miranda, W. López-Benítez, C.A. Jiménez-Bernardino
}

Introducción. Durante los estudios de especialidad el médico residente afronta niveles de estrés superiores a la población general afectándolo profesional y personalmente, por lo que cuestionamos por qué los estudios de residencia modifican la calidad de vida de los médicos residentes que los cursan. Sujetos y métodos. Se realizó un estudio descriptivo, comparativo, entre febrero de 2006 y febrero de 2007. Se aplicó el módulo central del cuestionario PECVEC a dos grupos de residentes: un grupo de residentes que iniciaba su especialidad y un año después, y a otro grupo que ya tenía más de un año realizando estudios de especialidad; se incluyeron residentes tanto de especialidades medicas como quirúrgicas que aceptaron participar. Los datos se capturaron en el programa SPSS v. 15. Se utilizó estadística descriptiva para variables sociodemográficas, $t$ de Student para comparar la calidad de vida entre residentes y especialidad cursada. La calidad de vida se clasificó como 'muy buena' de 3,1 a 4, 'buena' de 2,1 a 3, 'regular' de 1,1 a 2 y 'mala' entre 0 y 1 . Resultados. En el primer grupo se incluyeron 54 residentes, sólo 45 residentes completaron las encuestas al ingreso y un año después, y se excluyó a 9 por no completar la segunda evaluación; el segundo grupo incluyó a 62 residentes de segundo y tercer año que tenían más de un año realizando estudios de especialidad. Se encontró que después de un año de especialidad en las seis escalas evaluadas se presentó un descenso de la calidad de vida, cayendo de 'muy buena' $(3,18)$ a 'buena' $(2,73), p<0,001$; sin embargo, al compararlos con el grupo de segundo y tercer año, en estos últimos ya no hubo descenso en su calidad de vida. Los parámetros más afectados fueron función física y estado de ánimo negativo; de acuerdo con el género se afectaron en la misma proporción, y la especialidad más afectada fue Urgencias Médicas. Conclusión. Durante los estudios de residencia disminuye la calidad de vida de los médicos residentes sólo durante el primer año, aunque se mantiene en rangos aceptables.
Palabras clave. Ansiedad. Burnout. Calidad de vida. Depresión. Residencia médica.

\section{Measurement of the quality \\ of life in the residents doctors}

Introduction. During specialty studies, the resident doctor faces higher stress levels than the normal population, affecting him/her professionally and personally, hence we ask the question: do the residency studies modify the quality of life of the resident doctors who are taking the specialty? Subjects and methods. A descriptive, comparative study was carried out in February 2006 and in February 2007. We applied the PECVEC questionnaire central module to two resident's groups: the first one, initiating their medical specialty and one year later; and the second one, residents with more than one year of specialty studies. We included residents from medical and surgical specialties that agreed to participate in this study. All data was captured on SPSS V. 15 for analysis descriptive statistics were used for socio-demographic variables, the Student's t for comparing the quality of life among residents and specialty. The quality of life was classified as 'very good' between 3.1 and 4, 'good'between 2.1 and 3, 'regular' between 1.1 and 2 and 'bad' between 0 and 1. Results. 54 residents were included in the first group, from which only 45 completed the questionnaires at the beginning and one year later. We excluded 9 residents because they didn't complete the second questionnaire. In the second group, we included 62 second and third year residents that had more than a year of specialty studies. We found that after one year of specialty, the 6 evaluation scales showed a decline in the quality of life, falling from a level considered as 'very good' (3.18) to 'good' (2.73) $p<0.001$. However, when we compared these results with the second group, this one didn't have a decline in the quality of life; the most affected parameters were: physical function and negative state of mind, with respect to
Hospital General Regional n. ${ }^{\circ} 46$ 'Lázaro Cárdenas'. Instituto Mexicano del Seguro Social. Guadalajara, Jalisco, México.

\section{Correspondencia Dr. Sergio Emilio Prieto Miranda. Hospital General Regional n. ${ }^{\circ} 46$ 'Lázaro Cárdenas'. Instituto Mexicano del Seguro Social. Avda. Lázaro Cárdenas, 2063. Col. Morelos. CP 44190. Guadalajara, Jalisco, México. \\ E-mail serpri@avantel.net}

Agradecimientos A la Sra. Laura Quiñones Hernández, por su contribución en la revisión inicial del manuscrito. 
gender both were affected in the same proportion. The most affected specialty was emergency medicine. Conclusion. During the residency studies, the resident's quality of life does in fact decline in the first year, but it keeps acceptable levels.

Key words. Anxiety. Burnout. Depression. Medical residency. Quality of life.

\section{Introducción}

Durante el desarrollo de las residencias médicas se han encontrado niveles de estrés superiores a los de la población en general, los factores comúnmente asociados a esto son sobrecarga de trabajo, deprivación de sueño, quejas por parte de los pacientes y sus familiares hacia los residentes, conocimientos médicos insuficientes, ambientes académicos poco estimulantes, un alto grado de competitividad por parte de los propios compañeros e incertidumbre respecto al futuro profesional así como aspectos socioculturales y financieros [1]. Sí sólo tomáramos en cuenta la deprivación de sueño esta se encuentra asociada a una predisposición de cometer más errores, lesiones, abuso de alcohol o drogas y en el peor de los escenarios han ocurrido suicidios por parte de los residentes [2]. Todo esto lo encontramos de manera más marcada dentro del primer año de los cursos que se refleja en lo que se conoce como 'la historia natural del primer año de residencia' y que se caracteriza por excitación inicial (un mes), inseguridad (dos meses), depresión (un mes), aburrimiento (dos meses) y por último euforia, confianza y capacidad profesional [3]. Todos estos factores también pueden llevar a los médicos residentes a presentar un estado de agotamiento o burnout, el cual se le considera como una entidad moderna, derivada de la exposición crónica de manera gradual y continuada al estrés, siendo los profesionales de la salud la población más vulnerable a desarrollarlo [4]. Esto afecta más a los médicos residentes jóvenes, los cuales son más propensos a sufrir este síndrome, mientras que los médicos mayores de 45 años tienen mayor propensión a presentar insatisfacción [5]; también se ha encontrado que un 6,4\% de los residentes nunca se encontraban satisfechos con haber seleccionado la carrera de medicina [6].

También la vida personal de los médicos se ve afectada, tomando en cuenta tres aspectos: suici- dio, divorcio y depresión. Éstos se presentan con mayor frecuencia entre los médicos en general que entre la población general. La mayoría de los estudios comunican que la tasa de suicidio entre los médicos se encuentra entre 28 y 40 por 100.000 y es más alta en comparación con la población general, que es de 12,3 por 100.000 , situándose con esto como la mayor tasa entre los profesionales [7]. De acuerdo con Siergrist, calidad de vida se define en términos de dos dimensiones básicas de la salud subjetiva: la dimensión del bienestar (humor, emociones positivas y negativas, motivaciones y cognición) y en segundo lugar una dimensión de igual importancia que es la capacidad de actuación del sujeto (habilidad para alcanzar objetivos definidos como propios o ajenos) [8]. El cuestionario PECVEC, tanto en su versión en inglés [9] como en su versión al español [10], ha demostrado ser una herramienta muy útil en la evaluación de calidad de vida tanto en sujetos sanos como en sujetos enfermos. Como vemos en estas definiciones los aspectos más destacados son el bienestar, la capacidad de funcionamiento, el estado psicológico y la salud física del sujeto; éstos son los renglones que más comúnmente se ven afectados durante los estudios de especialidad, es decir, la calidad de vida. Sin embargo, y a pesar de esto, poco se ha investigado sobre este fenómeno. Nuestro objetivo fue medir la calidad de vida que tienen los médicos residentes a su ingreso en los cursos de especialidad y después de un año o más en el Hospital General Regional n. ${ }^{\circ} 46$ 'Lázaro Cárdenas' del Instituto Mexicano del Seguro Social, en Guadalajara Jalisco, México.

\section{Sujetos y métodos}

Entre el periodo de febrero de 2006 a febrero de 2007 se realizó un estudio descriptivo-comparativo a través de encuesta, previa aprobación por el Comité de Investigación y Ética de la unidad y consentimiento informado a los residentes de nuevo ingreso y de grados superiores tanto de las ramas médicas como quirúrgicas del Hospital General Regional n. 46 'Lázaro Cárdenas' del Instituto Mexicano del Seguro Social. El tipo de muestreo fue no probabilístico por conveniencia, la encuesta fue aplicada a dos grupos; un primer grupo de residentes a su ingreso y un año después, $\mathrm{y}$ un segundo grupo de residentes de segundo y 
Tabla I. Características sociodemográficas.

\begin{tabular}{|c|c|c|c|c|c|c|}
\hline & \multicolumn{2}{|c|}{$\begin{array}{c}\text { Residentes } \\
\text { de primer año }\end{array}$} & \multicolumn{2}{|c|}{$\begin{array}{c}\text { Residentes } \\
\text { de segundo año }\end{array}$} & \multicolumn{2}{|c|}{$\begin{array}{l}\text { Residentes } \\
\text { de tercer año }\end{array}$} \\
\hline Edad media & \multicolumn{2}{|c|}{$26,4 \pm 1,9$ años } & \multicolumn{2}{|c|}{$28,5 \pm 2,8$ años } & \multicolumn{2}{|c|}{$29,4 \pm 1,8$ años } \\
\hline Femenino & 19 & $42 \%$ & 30 & $64 \%$ & 9 & $60 \%$ \\
\hline Masculino & 26 & $58 \%$ & 17 & $36 \%$ & 6 & $40 \%$ \\
\hline Total & 45 & $100 \%$ & 47 & $100 \%$ & 15 & $100 \%$ \\
\hline Casados & 5 & $11 \%$ & 15 & $32 \%$ & 9 & $60 \%$ \\
\hline Solteros & 39 & $87 \%$ & 31 & $66 \%$ & 5 & $33 \%$ \\
\hline Divorciados & 1 & $2 \%$ & 1 & $2 \%$ & 1 & $7 \%$ \\
\hline Total & 45 & $100 \%$ & 47 & $100 \%$ & 15 & $100 \%$ \\
\hline Especialidad médica & 37 & $82 \%$ & 12 & $26 \%$ & 0 & $0 \%$ \\
\hline Especialidad quirúrgica & 8 & $18 \%$ & 35 & $74 \%$ & 15 & $100 \%$ \\
\hline
\end{tabular}

tercer año que ya se encontraban realizando su especialidad. Este grupo sólo fue encuestado en una ocasión con el fin de compararlos con el grupo inicial. Se incluyeron siete especialidades, tanto del área médica como quirúrgica, que aceptaron participar. Se excluyeron aquellos médicos que no desearon participar y los cuestionarios con menos del 90\% de las respuestas. La herramienta utilizada fue el cuestionario 'Perfil de calidad de vida en enfermos crónicos' (PECVEC) previamente validado al español [11]. Se utilizó el módulo central de este cuestionario, que consta de seis escalas: capacidad física, función psicológica, estado de ánimo positivo, estado de ánimo negativo, funcionamiento social, bienestar social, así como variables sociodemográficas (estado civil, sexo, edad), y preguntas adicionales donde se incluyeron apoyo social en la familia o en el círculo de amigos, acontecimientos vitales influyentes próximos o lejanos, problemas agudos en la última semana, y deterioro en la función sexual. En forma adicional se preguntó la especialidad de cada uno de los participantes. En cuanto al nivel de calidad de vida se consideró 'mala' si la puntuación media de todas las escalas estaba de 0 a 1, 'regular' de 1,1 a 2, 'buena' de 2,1 a 3 y 'muy buena' de 3,1 a 4. Se consideró mejoría en el nivel de la calidad de vida los casos que presentaron mejores puntuaciones estadísticamente significativas en dos de las seis escalas, así como variación en dos escalas más en sentido positivo. De igual manera se consideró deterioro en la calidad de vida cuando empeoraron dos de las seis escalas de manera significativa y variación en dos escalas más en sentido negativo. El análisis estadístico se realizó con el programa SPSS v. 15 a través de estadística descriptiva para las variables sociodemográficas y $t$ de Student para comparar la calidad de vida entre residentes y especialidad cursada. Se consideró $p$ significativa si fue $<0,05$.

\section{Resultados}

Se completaron 161 encuestas, en el primer grupo se incluyeron 54 residentes, aunque sólo 45 resi- 
Tabla II. Calidad de vida en médicos residentes.

\begin{tabular}{lcccc}
\hline Escala & $\begin{array}{c}\text { Inicio de residencia } \\
\text { (media } \pm \text { DE) }\end{array}$ & $\begin{array}{c}\text { Al año de residencia } \\
\text { (media } \pm \text { DE) }\end{array}$ & $t$ & $p$ \\
\hline Función física & $3,18 \pm 0,54$ & $2,73 \pm 0,60$ & $-3,698$ & 0,001 \\
\hline Función psicológica & $3,19 \pm 0,59$ & $2,93 \pm 0,61$ & $-2,830$ & 0,034 \\
\hline Estado de ánimo positivo & $2,84 \pm 0,69$ & $2,48 \pm 0,61$ & $-2,761$ & 0,008 \\
\hline Estado de ánimo negativo & $3,36 \pm 0,55$ & $2,94 \pm 0,64$ & $-3,626$ & 0,001 \\
\hline Función social & $3,01 \pm 0,69$ & $2,68 \pm 0,72$ & $-2,537$ & 0,015 \\
\hline Bienestar social & $3,29 \pm 0,61$ & $3,10 \pm 0,63$ & $-1,638$ & 0,109 \\
\hline
\end{tabular}

dentes completaron las encuestas al ingreso y un año después, y se excluyeron 9 residentes que no aceptaron participar al final del primer año o contestaron en forma incompleta el cuestionario. En el segundo grupo se incluyeron 62 residentes de segundo y tercer año que se encontraban realizando sus estudios de especialidad para compararlos con el grupo inicial. Las características generales de ambos grupos se muestran en la tabla I.

La fiabilidad de la prueba se determinó a través del coeficiente alfa de Cronbach, resultando con valores de bueno a excelente $(0,722-0,880)$.

Se realizó la comparación de los residentes del primer grupo al inicio y al final del primer año de su especialidad. Se observó un descenso en la puntuación del nivel de calidad de vida después de un año de exposición a los estudios de especialidad en cinco de las seis escalas valoradas, descendiendo por ejemplo en función física del nivel de 'muy bueno' (media de 3,18 ) a un nivel de 'bueno' (media de 2,73), $p=0,001$ (Tabla II).

Al comparar las puntuaciones de los médicos residentes al inicio y un año después entre las diferentes especialidades encontramos disminución de calidad de vida en las especialidades de medicina interna, medicina del trabajo y medicina de urgencias. Las áreas de mayor deterioro fueron función física y estado de ánimo negativo (Tabla III).

Al evaluar la calidad de vida de cada especialidad en forma global en sus seis escalas del cues- tionario, confirmamos el descenso en la calidad de vida de las especialidades arriba señaladas, con tendencia a afectar más a especialidades del área medica que a la quirúrgica (Tabla IV).

En relación al genero se afectaron tanto hombres (media: $-0,42692$; IC 95\% $=-0,75823$ a $-0,09561 ; p=0,014$ ) como mujeres (media: $-0,37895$; IC $95 \%=-0,72214$ a $-0,03578 ; p=$ $0,032)$. En el caso de las mujeres se afectaron más las escalas de capacidad física, estado de animo positivo y negativo, y en los hombres, la capacidad física y el estado de ánimo negativo.

Al comparar a los residentes solteros con los casados, ambos grupos mostraron descenso en su calidad de vida al final del primer año de residencia en todas las escalas, excepto en bienestar social en el caso de los solteros $(p=0,001)$; por el contrario, los casados mostraron mayor descenso en bienestar social.

Con el fin de demostrar si este descenso en la calidad de vida continuaba modificándose en sentido positivo o negativo en residentes con mayores años de exposición a los estudios de especialidad, se invitó a participar a 62 residentes de segundo y tercer año, a los cuales sólo se les aplicó en una ocasión la encuesta PECVEC. Se encontró que este grupo presentó resultados similares a los residentes que ya tenían un año de realizar sus estudios de especialidad, sin mayor descenso significativo en su calidad de vida, aunque tampoco mostraron mejoría en ésta (Tabla V). 
Tabla III. Calidad de vida de médicos residentes por especialidad.

\begin{tabular}{|c|c|c|c|c|c|c|c|c|c|c|c|c|}
\hline \multicolumn{2}{|c|}{ Ginecología } & \multicolumn{2}{|c|}{ Pediatría } & \multicolumn{2}{|c|}{ Cirugía } & \multicolumn{2}{|c|}{$\begin{array}{l}\text { Medicina } \\
\text { interna }\end{array}$} & \multicolumn{2}{|c|}{ Urgencias } & \multicolumn{2}{|c|}{$\begin{array}{l}\text { Aneste- } \\
\text { siología }\end{array}$} & $\begin{array}{c}\text { Medicina } \\
\text { del trabajo }\end{array}$ \\
\hline Media & $p$ & Media & $p$ & Media & $p$ & Media & $p$ & Media & $p$ & Media & $p$ & Media \\
\hline
\end{tabular}

Capacidad física

\begin{tabular}{|c|c|c|c|c|c|c|c|c|c|c|c|c|c|c|}
\hline Inicial & 3,31 & 0,461 & 3,125 & 0,997 & 3,06 & 0,208 & 3,48 & 0,006 & 3,16 & 0,010 & 2,95 & 0,562 & 3,00 & 0,014 \\
\hline $\begin{array}{l}1 \text { año } \\
\text { después }\end{array}$ & 3,00 & & 3,125 & & 2,53 & & 2,85 & & 2,47 & & 2,65 & & 2,75 & \\
\hline \multicolumn{15}{|l|}{$\begin{array}{l}\text { Función } \\
\text { psicológica }\end{array}$} \\
\hline Inicial & 3,12 & 0,815 & 3,0625 & 0,616 & 2,84 & 0,924 & 3,33 & 0,128 & 3,35 & 0,006 & 3,06 & 0,551 & 3,21 & 0,164 \\
\hline $\begin{array}{l}1 \text { año } \\
\text { después }\end{array}$ & 3,03 & & 3,3125 & & 2,81 & & 3,00 & & 2,60 & & 2,75 & & 3,23 & \\
\hline \multicolumn{15}{|c|}{$\begin{array}{l}\text { Estado de } \\
\text { ánimo positivo }\end{array}$} \\
\hline Inicial & 2,80 & 0,761 & 2,85 & 0,732 & 2,35 & 1 & 3,28 & 0,001 & 2,76 & 0,015 & 2,70 & 0,518 & 2,80 & 0,120 \\
\hline $\begin{array}{l}1 \text { año } \\
\text { después }\end{array}$ & 2,75 & & 2,6 & & 2,35 & & 2,66 & & 2,04 & & 2,40 & & 2,80 & \\
\hline
\end{tabular}

\section{Estado de}

ánimo negativo

\begin{tabular}{|c|c|c|c|c|c|c|c|c|c|c|c|c|c|c|}
\hline Inicial & 3,53 & 0,618 & 3,1875 & 0,659 & 2,81 & 0,493 & 3,57 & 0,006 & 3,62 & 0,002 & 3,08 & 0,507 & 3,23 & 0,028 \\
\hline $\begin{array}{l}1 \text { año } \\
\text { después }\end{array}$ & 3,43 & & 3,40625 & & 2,5 & & 2,95 & & 2,77 & & 2,77 & & 3,01 & \\
\hline
\end{tabular}

\section{Función social}

\begin{tabular}{|c|c|c|c|c|c|c|c|c|c|c|c|c|c|c|}
\hline Inicial & 3,04 & 0,602 & 3 & 1,000 & 2,83 & 0,424 & 3,18 & 0,100 & 3,11 & 0,012 & 2,77 & 0,358 & 2,95 & 0,126 \\
\hline $\begin{array}{l}1 \text { año } \\
\text { después }\end{array}$ & 2,87 & & 3 & & 2,29 & & 2,88 & & 2,38 & & 2,38 & & 3,04 & \\
\hline
\end{tabular}

Bienestar social

\begin{tabular}{|c|c|c|c|c|c|c|c|c|c|c|c|c|c|c|}
\hline Inicial & 3,30 & 0,308 & 3,35 & 0,670 & 2,80 & 0,576 & 3,46 & 0,358 & 3,28 & 0,041 & 3,36 & 0,457 & 3,08 & 0,181 \\
\hline $\begin{array}{l}1 \text { año } \\
\text { después }\end{array}$ & 3,50 & & 3,55 & & & & 3,26 & & 2,78 & & 3,13 & & 3,02 & \\
\hline
\end{tabular}

En lo referente a las preguntas adicionales encontramos que el $100 \%$ de los médicos residentes de ambos grupos consideran tener un adecuado apoyo social, mientras que en el apar- tado de acontecimientos vitales próximos o lejanos se observó que la mayoría de los médicos residentes respondió afirmativamente, siendo la razón más frecuente el cambio de domicilio e 
Tabla IV. Calidad de vida en médicos residentes agrupados por categorías.

\begin{tabular}{|c|c|c|c|c|c|c|c|}
\hline & $\begin{array}{c}\text { Nivel de calidad } \\
\text { de vida }\end{array}$ & Media & $\begin{array}{c}\text { Diferencia } \\
\text { entre medias }\end{array}$ & $\pm \mathrm{DE}$ & IC $95 \%$ & $t$ & $p$ \\
\hline \multirow{2}{*}{ Ginecología } & Inicial & 3,18 & $-0,0875$ & 0,34 & $\begin{array}{c}-0,836 \mathrm{a} \\
0,661\end{array}$ & $-0,372$ & 0,735 \\
\hline & 1 año después & 3,09 & & & & & \\
\hline \multirow{2}{*}{ Pediatría } & Inicial & 3,09 & 0,07 & 0,40 & $\begin{array}{c}-1,672 \mathrm{a} \\
1,812\end{array}$ & 0,128 & 0,906 \\
\hline & 1 año después & 3,16 & & & & & \\
\hline \multirow{2}{*}{ Cirugía } & Inicial & 2,83 & $-0,2875$ & 0,73 & $\begin{array}{c}-1,5198 a \\
0,948\end{array}$ & $-0,742$ & 0,512 \\
\hline & 1 año después & 2,54 & & & & & \\
\hline \multirow{2}{*}{ Medicina interna } & Inicial & 3,38 & $-0,454$ & 0,36 & $\begin{array}{c}-0,7199 a \\
-0,1881\end{array}$ & $-3,862$ & 0,004 \\
\hline & 1 año después & 2,93 & & & & & \\
\hline \multirow{2}{*}{ Urgencias } & Inicial & 3,21 & $-0,705$ & 0,50 & $\begin{array}{c}-1,1029 a \\
-0,3071\end{array}$ & $-4,008$ & 0,003 \\
\hline & 1 año después & 2,5 & & & & & \\
\hline \multirow{2}{*}{ Anestesiología } & Inicial & 2,99 & $-0,31167$ & 0,55 & $\begin{array}{c}-1,3125 a \\
0,6892\end{array}$ & $-0,8$ & 0,46 \\
\hline & 1 año después & 2,68 & & & & & \\
\hline \multirow{2}{*}{ Medicina del trabajo } & Inicial & 3,04 & $-0,305$ & 0,73 & $\begin{array}{c}-0,6017 a \\
-0,0083\end{array}$ & $-2,117$ & 0,044 \\
\hline & 1 año después & 2,97 & & & & & \\
\hline
\end{tabular}

inicio de la especialidad con un $78 \%$. Respecto a los médicos residentes con más de un año de estudios de especialidad solamente respondió afirmativamente una tercera parte, siendo los eventos más mencionados muerte o enfermedad de un familiar y término de relaciones interpersonales. En cuanto a presentar problemas agudos en la última semana, fue más frecuente en los médicos residentes que tenían más de un año de estudios de especialidad que en los que recién ingresaban, afectando también la función sexual.

\section{Discusión}

Existen en la literatura mundial escasos trabajos que aborden la problemática de la calidad de vida dentro de los médicos residentes; sin embargo, los existentes coinciden en una disminución de la misma. En general, el aspecto con mayor deterioro después de año de exposición a los estudios de especialidad es la función física, en esto puede ser clave la deprivación de sueño que de manera aguda y crónica sufren los residentes. Se ha demostrado que los médicos residentes antes 
Tabla V. Comparación de calidad de vida en médicos residentes de diferente año.

\begin{tabular}{lccccccc}
\hline & $\begin{array}{c}\text { Residentes } \\
\text { al inicio }\end{array}$ & \multicolumn{2}{c}{$\begin{array}{c}\text { Residentes } \\
\text { primer año }\end{array}$} & $\begin{array}{c}\text { Residentes de } \\
\text { segundo año }\end{array}$ & $\begin{array}{c}\text { Residentes de } \\
\text { tercer año }\end{array}$ \\
\hline Media & Media & $p$ & Media & $p$ & Media & $p$ \\
\hline Capacidad física & 3,16 & 2,69 & 0,001 & 2,66 & 0,914 & 2,55 & 0,409 \\
\hline Función psicológica & 3,2 & 2,87 & 0,037 & 2,77 & 0,485 & 2,6 & 0,268 \\
\hline Estado de ánimo positivo & 2,92 & 2,49 & 0,01 & 2,38 & 0,849 & 2,34 & 0,439 \\
\hline Estado de ánimo negativo & 3,37 & 2,97 & 0,001 & 2,79 & 0,742 & 2,8 & 0,425 \\
\hline Función social & 3,05 & 2,64 & 0,03 & 2,58 & 0,535 & 2,43 & 0,404 \\
\hline Bienestar social & 3,28 & 3,11 & 0,157 & 2,99 & 0,595 & 2,98 & 0,805 \\
\hline Total de residentes & 45 & 45 & & 47 & & 15 & \\
\hline
\end{tabular}

de una guardia duermen un promedio de $6,47 \mathrm{~h}$ y posteriormente a una guardia, $14 \mathrm{~h}$ en los siguientes dos días, cuando lo recomendado en adultos de las edades de los médicos residentes que cursan especialidades es de 8 a 10 h antes de una deprivación aguda de sueño y 16 h con posterioridad [12]. Las puntuaciones medias de las seis escalas valoradas por el PECVEC obtenidas en nuestro estudio son mayores a las encontradas en un estudio similar en médicos familiares en Argentina por Albanesi [8], inclusive después de un año de exposición a los estudios de especialidad en el caso de nuestro estudio. En ambos casos, la mayor afectación se da en el estado de ánimo positivo, presentando en el estudio realizado en Argentina una puntuación media de 1,98 para esta escala y en nuestro estudio de 2,49 , lo cual podría interpretarse como el preludio de un estado de decepción y depresión, fenómenos muy frecuentes durante el desarrollo de los estudios de residencia. Otra coincidencia con este estudio es en la escala de bienestar social; fue la mejor calificada, con puntuaciones medias de 3,2 y 3,1 respectivamente. A pesar del descenso podemos inferir que el médico residente conserva cierta satisfacción hacia su entorno al estar realizando sus estudios de posgrado y en esto influye el entorno laboral donde se está desarrollando el curso de especialización. Al comparar la calidad de vida de acuerdo con el género de los médicos residentes encontramos diferencias entre nuestro estudio y el realizado por Albanesi, ya que en este último se observaron diferencias significativas entre los géneros, siendo más afectado el sexo femenino, mientras que en nuestro estudio no se establecieron diferencias significativas entre los géneros. Sin embargo, en ambos estudios, las escalas de función física y estado de ánimo positivo fueron las más afectadas en el caso del sexo femenino [8]. De las variables estudiadas, la especialidad cursada en las que se destacaron más las diferencias fueron medicina de urgencias, medicina interna y, en menor proporción, los residentes de medicina del trabajo; resultados similares se encontraron en dos estudios, uno en el que se estudió depresión y ansiedad durante el primer año de la residencia, donde se observó mayor nivel de depresión y ansiedad en los médicos residentes que rotaban por los servicios de urgencias, afectándose hasta un 6,9\% de los estudiados, sobre todo durante su primera rotación [3]. En el segundo estudio se evaluó la incidencia de burnout, en el cual se encontró que un $61,5 \%$ de los residentes de urgencias y medicina interna 
presentaron dicho síndrome [13]. Quizás esto se deba a los niveles de estrés, al tipo de pacientes que se manejan en esta área, además del hecho de que los residentes de estás áreas en ocasiones deben enfrentarse a situaciones desconocidas sin el apoyo de médicos de mayor jerarquía, o por la falta de cobertura de los médicos adscritos de estas áreas, particularmente en nuestros hospitales de seguridad social, donde existe carencia de estos especialistas.

Al comparar el nivel de calidad de vida de los residentes de acuerdo con su estado civil no encontramos diferencias significativas, presentando ambos grupos en el primer año un descenso en su calidad de vida, excepto en bienestar social, que se afectó menos en solteros y sorpresivamente más en los casados. Coincide con lo encontrado en otro estudio en el que se buscó el grado de satisfacción personal con la especialidad, y se observó que los médicos residentes casados con hijos presentaron un grado mayor de insatisfacción con su trabajo actual [5]; sin embargo, esto contrasta con otros estudios en los que se encontraron diferencias a favor de los casados, y de que el ser soltero fuera un factor de riesgo para presentar síndrome de burnout [4], mientras que en otro estudio se observó que los médicos residentes con hijos mostraban menos sentimientos de depresión y cinismo que los médicos residentes sin hijos [14]. Estas diferencias respecto a nuestro estudio y lo comunicado por otros autores puede ser resultado de que muchos residentes, al iniciar sus estudios de especialización, se encuentran aún solteros, como sucedió con nuestra muestra estudiada. Como vemos, los estudios de especialidad parecen afectar de manera negativa a los médicos que los cursan sólo en el primer año de la residencia, pero si tomamos en cuenta que su calidad de vida basal es muy buena y desciende a buena en este primer año, y que mantiene este mismo nivel los siguientes años, podríamos inferir que sólo se trate de un proceso de adaptación, o que se pueda relacionar también con otros factores, como insatisfacción por su carrera -como ya habían descrito Sepúlveda y Vildosola [5]-, sobre todo en los primeros años de especialidad. Por ello debemos plantearnos si los estudios de residencia pueden ser exitosos cuando los médicos que los cursan presentan depresión, ansiedad, cinismo, deshumanización y disminución de su nivel de calidad de vida y cómo esto puede reflejarse en la atención medi- ca que deben prestar estos residentes. ¿Se puede revertir esta situación? En un estudio llevado a cabo en 2005, en el cual se preguntó a los médicos encargados de la enseñanza de los residentes acerca de qué efectos había tenido la reducción de los horarios de trabajo de los residentes, se encontró una percepción de mejoría en el aspecto del bienestar, incluido el nivel de fatiga (85\%) y el equilibrio entre la vida personal y profesional (81\%), así como una disminución en la incidencia del síndrome de burnout dentro de los residentes. Resultados similares se encontraron en un estudio de la Universidad de California, en el cual se observó una mejoría en la calidad de vida de la mayoría de los residentes $[15,16]$. Sin embargo, en los dos estudios se encontró que el tiempo libre no se utiliza en actividades educativas, además de encontrarse efectos negativos en cuanto al cuidado de los pacientes y el profesionalismo de los residentes, por lo que aún queda pendiente hallar un equilibrio de cómo mejorar la calidad de vida de los residentes sin que se vea afectada su formación y la atención y salud de los pacientes que están a cargo de este personal en formación.

Dentro de las preguntas adicionales llama la atención que los médicos de reciente ingreso consideren su cambio de domicilio como algo trascendental, así como el inicio de la especialidad. Es común, incluso hasta posgrado, que los estudiantes realicen sus estudios básicos conviviendo aún en el núcleo familiar en México, por lo que la asignación de sus estudios de residencia a una plaza diferente es tomado como un evento trascendental. El inicio de los estudios de posgrado sí representa un hecho aún mucho mayor, dado que en México sólo un 30\% de los médicos generales pueden aspirar a realizar estudios de posgrado debido a la falta de espacios, nivel educativo heterogéneo entre las diferentes universidades que forman médicos y el nivel de complejidad del mismo examen nacional. Nuestro hospital es el mayor del Occidente de México que presta servicios de seguridad social de segundo nivel de atención, con más de 425 camas censables y con una plantilla de 107 residentes distribuidos en las siete especialidades estudiadas. Las cargas de trabajo de los residentes de especialidades de medicina de urgencias y medicina interna podrían explicar el descenso en su calidad de vida al tener hasta $95 \mathrm{~h}$ de trabajo semanal. Sin embargo, es de notar que este descenso en 
su calidad de vida se mantiene en niveles aceptables incluso dos o tres años después de estar realizando sus estudios de especialidad, como han demostrado otros autores [1-3]. Por el contrario, los residentes de medicina del trabajo no tienen estas cargas de trabajo semanal y mostraron tendencia a disminuir su calidad de vida, por lo que se requieren estudios controlados y más amplios para confirmar nuestros resultados.

\section{Bibliografía}

1. Luthy C, Perrier A, Perrin E, Cedraschi C, Allaz AF. Exploring the major difficulties perceived by residents in training: a pilot study. Swiss Med Wkly 2004; 134: 612-7.

2. Cohen JS, Patten S. Well-being in residency training: a survey examining resident physician satisfaction both within and outside of residency training and mental health in Alberta. BMC Med Educ 2005; 5: 21.

3. Peterlini M, Tiberio IF, Saadeh A, Pereira JC, Martins MA. Anxiety and depression in the first year of medical residency training. Med Educ 2002; 36: 66-72.

4. Aranda-Beltrán C, Pando-Moreno M, Salazar-Estrada JG, Torres-López TM, Aldrete-Rodríguez MG, Pérez-Reyes MB. Síndrome de burnout en médicos familiares del Instituto Mejicano del Seguro Social, Guadalajara, México. Rev Cubana Salud Pública 2005; 31: 1-9.

5. Sepúlveda-Vildosola AC, Flores-Pulido AA, López-Fuentes G, López-Aguilar E. Insatisfacción durante la residencia de pediatría. Prevalencia y factores asociados. Rev Med Inst Mex Seguro Soc 2006; 44: 423-32.

6. Miller MN, McGowen KR. The painful truth: physicians are not invincible. South Med J 2000; 93: 966-73.
7. The NSDUH Report. October 2007. Depression among adults employed full-time, by occupational category. URL: http://www.oas.samhsa.gov/2k7/depression/occupation. htm. [12.02.2008].

8. Albanesi de Nasetta $S$, Nasetta P. Residentes médicos y calidad de vida. Vertex 2005; 16: 245-50.

9. Simon E, Scwartz M, Roos J, Dragano N, Geraedts M, Siegrist J, et al. Evaluation of quality of life and description of the sociodemographic state in adolescent and young adult patients with phenylketonuria (PKU). Health Qual Life Outcomes 2008; 6: 25.

10. Fernández-Lopez JA, Rancaño-Mejía I, Hernández-Mejía R. Propiedades psicométricas de la versión española del cuestionario PECVEC de perfil de calidad de vida en enfermos crónicos. Psicothema 1999; 11: 293-303

11. Fernández-López JA, Siegrist J, Hernández-Mejía R, Broer M, Cueto-Espinar A. Evaluación de la equivalencia transcultural de la versión española del perfil de calidad de vida para enfermos crónicos (PECVEC). Med Clin (Barc) 1997; 109: 245-50.

12. Arora VM, Georgitis E, Woodruff JN, Humphrey HJ, Meltzer D. Improving sleep hygiene of medical interns: can the sleep, alertness, and fatigue education in residency program help? Arch Intern Med 2007; 167: 1738-44.

13. López-Morales A, González-Velázquez F, Morales-Guzmán MI, Espinoza-Martínez CE. Síndrome de burnout en residentes con jornadas laborales prolongadas. Rev Med Inst Mex Seguro Soc 2007; 45 :233-42.

14. Collier VU, McCue JD, Markus A, Smith L. Stress in medical residency: status quo after a decade of reform. Ann Intern Med 2002; 136: 384-90.

15. Reed DA, Levine RB, Miller RG, Ashar BH, Rice TN. Effect of residency duty-hour limits: views of key clinical faculty. Arch Intern Med 2007; 167: 1453-5.

16. Yoon HH. Adapting to duty-hour limits -four years on. N Engl J Med 2007; 356: 2668-70. 